\title{
Individualisierung der antihypertensiven Therapie bei Patienten mit Diabetes mellitus. Leitlinie der Österreichischen Diabetes Gesellschaft (Update 2019)
}

\author{
Guntram Schernthaner · Christoph H. Saely · Gerit-Holger Schernthaner · Bruno Watschinger · Heinz Drexel
}

(c) Der/die Autor(en) 2019

\begin{abstract}
Zusammenfassung Hypertonie ist eine sehr häufige Komorbidität bei Patienten mit Diabetes mellitus, die - wenn unzureichend behandelt - signifikant zur erhöhten Mortalität und zum Auftreten von mikrovaskulären und makrovaskulären Komplikationen beiträgt. Eine Individualisierung der Blutdruckzielwerte in Abhängigkeit vom Patientenalter und vom Vorliegen bestimmter vaskulärer Komplikationen wird heute weltweit diskutiert. Blutdruckzielwerte um $130 / 80 \mathrm{~mm} \mathrm{Hg}$ waren in den Studien mit der geringsten Ereignisrate an Komplikationen assoziiert. ACEHemmer und Angiotensin-Rezeptorblocker sollen in der Hypertonie-Therapie bei Patienten mit Diabetes mellitus bevorzugt werden. Calciumantagonisten und Diuretika sind in der Second-Line-Therapie oft notwendig. Nach Erreichung der Zielwerte muss die antihypertensive Therapie fortgeführt werden, wobei regelmäßige Blutdruckmessungen durch die Patienten für die Optimierung der Blutdruckeinstellung sehr hilfreich sind. Neuere Antidiabetika wie SGLT2-Inhi-
\end{abstract}

bitoren oder GLP1-Rezeptoragonisten haben tragen ebenfalls zur Blutdrucksenkung bei.

Schlüsselwörter Hypertonie - Diabetes · Blutdruckzielwerte · Individualisierung • Vaskuläre Komplikationen

\section{Individualising antihypertensive therapy in patients with diabetes. A guideline by the Austrian Diabetes Association (Update 2019)}

Summary Hypertension is one of the most important comorbidities of diabetes, contributing significantly to death and leads to macrovascular and microvascular complications. When assessing the medical priorities for patients with diabetes, treating hypertension should be a primary consideration. In the present review practical approaches to hypertension in diabetes, including individualized targets for preventing specific complications are discussed according to cur-

G. Schernthaner $(\bowtie)$

1. Medizinische Abteilung, Krankenanstalt Rudolfstiftung, Juchgasse 25, 1030 Wien, Österreich

guntram.schernthaner@meduniwien.ac.at;

guntram@schernthaner.eu

C. H. Saely

Abteilung für Innere Medizin I, Akademisches

Lehrkrankenhaus Feldkirch, Feldkirch, Österreich

G.-H. Schernthaner

Klinische Abteilung für Angiologie, Universitätsklinik für Innere Medizin II, Medizinische Universität Wien, Wien, Österreich

B. Watschinger

Klinische Abteilung für Nephrologie und Dialyse,

Universitätsklinik für Innere Medizin III, Medizinische

Universität Wien, Wien, Österreich 
rent studies and guidelines. According to recent studies, blood pressure values of about $130 / 80 \mathrm{~mm} \mathrm{Hg}$ are associated with the best outcome. Angiotensin converting enzyme inhibitors and angiotensin receptor blockers are the most effective drugs for treating hypertension in diabetes. Calcium antagonists or diuretics are acceptable as second-line agents. Once the target is achieved, antihypertensive drugs should be continued. Newer antidiabetic medications such as SGLT-2-inhibitors or GLP1-receptor agonists have also antihypertensive effects.

Keywords Hypertension - Diabetes - Target values · Individualisation · Vascular complications

\section{Definition}

Eine arterielle Hypertonie soll dann diagnostiziert werden, wenn der Blutdruck bei wiederholten Messungen an verschiedenen Tagen $\geq 140 / 90$ mm Hg liegt. Rezent wurden in den USA niedrigere Grenzwerte für die Diagnose einer Hypertonie vorgeschlagen [1]; aufgrund der fraglichen therapeutischen Konsequenz wird diese neue Definition allerdings kontrovers diskutiert [2-4]. Die vorliegenden österreichischen Leitlinien [5, 6] definieren eine arterielle Hypertonie bei Diabetes mellitus übereinstimmend mit mehreren internationalen Leitlinien als einen Blutdruck $\geq 140 / 90$ mm Hg.

\section{Epidemiologie und Screening}

Die arterielle Hypertonie ist eine sehr häufige Komorbidität sowohl bei Patienten mit Typ 2 als auch bei Patienten mit Typ 1 Diabetes mellitus, und ein erhöhter Blutdruck $\geq 140 \mathrm{~mm} \mathrm{Hg}$ systolisch und/oder $\geq 90 \mathrm{~mm}$ $\mathrm{Hg}$ diastolisch findet sich in Abhängigkeit von Diabetesdauer und Ausmaß der Adipositas in 50-60\% aller Patienten [7]. Der Blutdruck sollte bei jeder Visite eines Patienten mit Diabetes kontrolliert werden. Hypertensive Patienten mit Diabetes sollten ihren Blutdruck auch zuhause selbst regelmäßig kontrollieren. Häufig geht die Hypertonie dem Diabetes zeitlich voraus und findet sich dabei assoziiert mit anderen Komponenten des Insulinresistenzsyndroms. Patienten mit Hypertonie haben ein 2,2-fach erhöhtes Risiko innerhalb von 5 Jahren einen Diabetes zu entwickeln. Sie sollen deshalb auf das Vorliegen einer Glukosestoffwechselstörung gescreent werden.

Im Vergleich zu nichtdiabetischen Patienten findet man insbesondere bei älteren Diabetes-Patienten häufig eine isolierte systolische Hypertonie. Das häufige Fehlen einer physiologischen Nachtabsenkung („Dipping“) geht mit einer Mikroalbuminurie bzw. Linksventrikelhypertrophie einher und ist ein ungünstigster Prädiktor für kardiovaskuläre Ereignisse.

\section{Lebensstilinterventionen zur Blutdrucksenkung}

Lebensstilmaßnahmen sind die Basis jeder antihypertensiven Therapie. Bereits bei Patienten mit Blutdruckwerten $>120 / 80 \mathrm{~mm} \mathrm{Hg}$ sollten eine Gewichtsreduktion bei Übergewicht oder Adipositas, eine Kochsalz-arme und Kalium-reiche Diät, der Verzicht auf übermäßigen Alkoholkonsum sowie körperliche Aktivität empfohlen werden. In der Initialphase der LOOK-AHEAD-Studie $(n=5145)$ senkte eine Gewichtsreduktion von zirka $8 \mathrm{~kg}$ nach einem Jahr bei übergewichtigen/adipösen Patienten mit Typ 2 Diabetes die Blutdruckwerte um 5/2 mm Hg signifikant [8]. Bei Patienten mit einem Gewichtsverlust $>15 \%$ betrug die systolische Blutdrucksenkung sogar $10 \mathrm{~mm}$ Hg [8].

\section{Individualisierung des Zielblutdruckes bei Patien- ten mit Diabetes mellitus?}

In epidemiologischen Studien fand sich ein enger $\mathrm{Zu}-$ sammenhang zwischen dem Auftreten von makrovaskulären Komplikationen wie koronare Herzkrankheit (KHK), Schlaganfall, peripherer arterieller Verschlusskrankheit (PAVK) sowie mikrovaskulären Komplikationen (diabetische Nephropathie und diabetische Retinopathie) und den erhobenen Blutdruckwerten [7]. Da in epidemiologischen Analysen das niedrigste Risiko bei Blutdruckwerten von 120/80 mm Hg beobachtet wurde [8, 9], glaubte man, dass möglichst niedrige Blutdruckwerte mittels antihypertensiver Therapiemaßnahmen bei Patienten zu erzielen wären. In der IDNT-Studie [10] wurde eindrucksvoll gezeigt, dass das renale Überleben bis unter $120 \mathrm{~mm} \mathrm{Hg}$ besser ist, dass allerdings die Mortalität bei $<121 \mathrm{~mm} \mathrm{Hg}$ systolisch dramatisch wieder ansteigt (J-Curve).

Bis 2007 wurde deshalb in allen Guidelines ein systolischer Zielblutdruck bei Patienten mit Diabetes mellitus unabhängig vom Alter $<130 \mathrm{~mm}$ Hg gefordert [11, 12], wobei für Patienten mit diabetischer Nephropathie sogar Zielwerte unter 120/75 mm Hg empfohlen wurden, obwohl Evidenz-basierte Interventionsstudien für diese Zielwerte nie vorlagen. In weiterer Folge publizierte Interventionsstudien bestätigten allerdings die Annahme eines generellen Vorteils sehr niedriger Blutdruckwerte nicht [13-16]. 2008 wurden in der ACCORD-Studie Nachteile einer zu niedrigen Blutdruckeinstellung ( $<120 \mathrm{~mm} \mathrm{Hg})$ nachgewiesen. Im Blutdruckarm der ACCORD-Studie wurden 4700 Patienten mit Typ 2 Diabetes entweder einer intensivierten Therapie (systolischer Blutdruck < $120 \mathrm{~mm} \mathrm{Hg}$ ) oder einem Standardtherapiearm (systolischer Blutdruck<140 mm Hg) zugeordnet [17]. Nach ca. 5 Jahren betrugen die Blutdruckwerte $119 \mathrm{~mm} \mathrm{Hg}$ versus $133 \mathrm{~mm}$ Hg. Durch die intensivierte Therapiestrategie wurde der primäre Endpunkt schwerer kardiovaskulärer Ereignisse nicht signifikant gesenkt, Schlaganfälle nahmen um $47 \% \mathrm{ab}$, während die Mortalität nicht beeinflusst wurde. Die intensivierte Therapie war al- 
lerdings mit schwerwiegenden Rhythmusstörungen, Hyperkaliämie, Arrhythmie und Hypotension assoziiert. Subsets der Patienten in ACCORD mit hohem kardiovaskulärem Risiko profitierten allerdings von einer aggressiven Blutdruckeinstellung [18, 19]. Ähnlich wie bei der Intensität der Glukoseeinstellung [20] scheint es also sinnvoll, die Intensität der antihypertensiven Therapie individuell auf den Patienten anzupassen [21].

Dabei muss aber vorsichtig vorgegangen werden. In einer Studie von Cooper-DeHoff et al. [22] fand sich etwa bei Patienten mit Diabetes und koronarer Herzerkrankung unter einer intensivierten Blutdrucksenkung eine Zunahme der Gesamtmortalität; bei Patienten mit systolischen Blutdruckwerten $<110$ mm Hg war die Mortalität im Vergleich zu Patienten mit systolischen Blutdruck-Werten von 125-130 mm Hg signifikant erhöht. Auch in der VADT-Studie fand sich eine signifikante Zunahme der kardiovaskulären Ereignisse, wenn die diastolischen Blutdruck-Werte $<70 \mathrm{~mm}$ Hg abgesenkt wurden [23].

\section{Heterogene Blutdruckzielwerte verschiedener Wissenschaftsgesellschaften}

Die aktuellen Leitlinien der American Diabetes Association (ADA) 2018 [24] fordern ein generelles Blutdruckziel von $<140 / 90 \mathrm{~mm} \mathrm{Hg}$, niedrigere Blutdruckziele $<130 / 80 \mathrm{~mm}$ Hg können bei hohem kardiovaskulärem Risiko erwogen werden, wenn das Erreichen dieser Werte adäquat vertragen wird. Die rezenten Guidelines der American College of Cardiology/American Heart Association empfehlen hingegen Blutdruckzielwerte von $<130 / 80 \mathrm{~mm} \mathrm{Hg}$ bei Patienten mit und ohne Diabetes mellitus [25]. Blutdruckzielwerte von $<130 / 80 \mathrm{~mm} \mathrm{Hg}$ werden aber auch von Kanadischen Experten [26], der IDF [27]; WHO; [28], UK-NICE [29] und von Japanischen Experten [30] empfohlen (Tab. 1). Die im September 2018 publizierten Guidelines der ESH/ESC empfehlen ebenfalls einen systolischen Blutdruckzielwert von $130 \mathrm{~mm} \mathrm{Hg}$ oder niedriger wenn toleriert, allerdings nicht unter

Tab. 1 Verschiedene Blutdruckziele verschiedener internationalen Fachgesellschaften

\begin{tabular}{|c|c|c|}
\hline Fachgesellschaft & Ziel & Primäre Medikamentenempfehlung \\
\hline ACC/AHA [25] & $130 / 80$ & ACEI/ARB \\
\hline CHEP [26] & $130 / 80$ & ACEI/ARB \\
\hline IDF [27] & $130 / 80$ & ACEI/ARB \\
\hline WHO [28] & $130 / 80$ & ACEI/ARB \\
\hline UK Nice [29] & $130 / 80$ & ACEI/ARB \\
\hline JSH [30] & $130 / 80$ & ACEI/ARB \\
\hline IGH [31] & $140 / 80$ & ACEI/ARB \\
\hline ESC/ESH [32] & $130 / 80$ & ACEI/ARB \\
\hline ADA [24] & $140 / 90$ & ACEI/ARB \\
\hline JNC-8P [33] & $140 / 90$ & ACEI/ARB \\
\hline
\end{tabular}

$120 \mathrm{~mm} \mathrm{Hg}$. Andererseits erlauben größtenteils ältere Guidelines auch höhere Zielwerte ([31, 33, 34]; Tab. 1).

\section{Warum ist das alles so kompliziert?}

Die unterschiedlichen Empfehlungen basieren darauf, dass die Studienlage hinsichtlich intensivierter Blutdrucksenkung komplex ist und widersprüchlich interpretiert wird, wobei dies insbesondere für die Studien ACCORD-BP (bei Diabetikern) [35] und SPRINT (bei Nichtdiabetikern) [36] gilt. In beiden Studien wurden im intensivierten Studienarm Blutdruckzielwerte $<120 / 80 \mathrm{~mm} \mathrm{Hg}$ verfolgt und mit den Ergebnissen der Kontrollkohorten (Zielwert $<140 / 80 \mathrm{~mm}$ $\mathrm{Hg}$ ) verglichen. Während in der ACCORD-BP-Studie der kardiovaskuläre Tod durch die intensivierte Blutdruckkontrolle nicht gesenkt wurde (HR 1,06; $95 \%$ CI $0,74-1,52)$, fand sich in der SPRINT $(0,57$; 0,38-0,85) Studie eine dramatische Senkung. Auch die Unterschiede in der Risikobeeinflussung für die Herzinsuffizienz waren kontrastierend: ACCORD-BP $(0,94 ; 0,70-1,26)$, und SPRINT $(0,62 ; 0,45-0,84)$.

Es ist unklar, ob die unterschiedlichen Studienergebnisse auf die differenten Populationen zurückzuführen sind oder ob auch das Studiendesign einen Einfluss hatte [37]. Auch die unterschiedliche Blutdruckmessung muss dabei bedacht werden. Bei der unbeobachteten automatisierten Messung in SPRINT sind insgesamt niedrigere Werte $\mathrm{zu}$ erwarten, die wahrscheinlich höheren in der Selbstmessung und bei der Ordinationsmessung entsprechen.

In der ACCORD-Studie wurde gleichzeitig auch der Effekt einer intensivierten Blutzuckerkontrolle (HbAlc-Zielwert<6,0\%) untersucht, wobei in der intensivierten Gruppe eine erhöhte kardiovaskuläre Mortalität beobachtet wurde [35]. In der Re-Analyse der ACCORD-Studie [38] fand sich im glykämischen Standardarm unter intensivierter Blutdrucksenkung eine signifikante Senkung von Schlaganfall (-39\%), der CV-Endpunkte (-33\%) und des Myokardinfarktes $(-37 \%)$. Mancia und Grassi [4] diskutieren einen potentiell negativen Effekt von schweren Hypoglykämien im intensivierten Blutzuckerarm auf den potentiell vorteilhaften Effekt der intensivierten Blutdruckeinstellung.

In einer rezenten gepoolten Analyse von ACCORDPB und SPRINT fanden sich ähnlich günstige Effekte auf kardiovaskuläre Endpunkte unter einer intensivierten Blutdruckkontrolle mit fehlender statistischer Heterogenität [19]. In einer weiteren gepoolten Sekundäranalyse von ACCORD-PB und SPRINT fanden sich hingegen warnende Hinweise, dass eine $\mathrm{zu}$ strikte Blutdruckeinstellung die Inzidenz der chronischen Niereninsuffizienz (CNI) steigern dürfte [39]. Bei Patienten ohne CNI vor Intervention $(n=4311$ in ACCORD; $n=6715$ in SPRINT), wurde der Effekt der systolischen Blutdrucksenkung $(13,9 \mathrm{~mm} \mathrm{Hg}$ in ACCORD und 15,2 mm $\mathrm{Hg}$ in SPRINT) auf einen Abfall der eGFR $>30 \%$ auf $<60 \mathrm{~mL} / \mathrm{min}$ per $1,73 \mathrm{~m}^{2}$ ) 
analysiert. Nach drei Jahren betrug die kumulative Inzidenz einer CNI in der ACCORD-Studie $10 \%$ im intensivierten Arm vs. 4,1\% im Standardarm; auch in der SPRINT-Studie war die Inzidenz einer CNI signifikant höher im intensivierten Arm - 3,5\% vs. 1,0\% im Standardarm. Eine Überwachung der Nierenfunktion unter intensivierter Blutdruckkontrolle ist daher sehr wichtig.

Eine rezente große Kohortenstudie [40] spricht für ein Blutdruckziel $<140 \mathrm{~mm} \mathrm{Hg}$ auch bei Patienten mit Typ 2 Diabetes ohne etablierte kardiovaskuläre Erkrankungen; niedrigere systolische Werte $<130$ oder $<120 \mathrm{~mm} \mathrm{Hg}$ waren mit keinem zusätzlichen kardiovaskulären Vorteil assoziiert. Aufschlussreich ist auch eine rezente retrospektive Kohorten-Studie aus Hongkong [40] bei 28.014 Patienten mit Typ 2 Diabetes ohne Vorliegen einer kardiovaskulären Erkrankung. Die Inzidenz einer kardiovaskulären Erkrankung nach einer mittleren Beobachtungszeit von 5 Jahren war nach den erreichten Blutdruckzielwerten deutlich unterschiedlich $<120 \mathrm{~mm} \mathrm{Hg}(15,3 \%)$, $<130$ mm Hg (9,1\%), <140 mm Hg (10,1\%) [40]. Patienten mit erreichten Blutdruck-werten $<120 \mathrm{~mm} \mathrm{Hg}$ hatten ein signifikant höheres Risiko als Patienten mit Werten $<130 \mathrm{~mm}$ Hg (Hazard Ratio (HR) 1,75 [95\% Confidence Intervall [CI]: 1,53-2,00]) oder $<140 \mathrm{~mm}$ Hg (HR 1,67 [95\% CI: 1,46-1,90]). Bei jüngeren Patienten ( $<65$ Jahre) war das Risiko allerdings signifikant geringer, wenn Blutdruckwerte $<130 \mathrm{~mm} \mathrm{Hg}$ erzielt wurden (HR 0,81 [95\% CI: 0,69-0,96]) im Vergleich zu Patienten mit Blutdruckwerten $<140 \mathrm{~mm} \mathrm{Hg}$ [40]. Bei älteren Patienten ( $>65$ Jahre) hingegen war kein Vorteil für Blutdruckwerte $<130 \mathrm{~mm} \mathrm{Hg} \mathrm{zu}$ beobachten [40].

Bezüglich der Zielwerte für den diastolischen Blutdruck besteht eine weitgehende Übereinstimmung der Expertenmeinung. In der HOT (Hypertension Optimal Treatment) Studie fand sich bei Typ 2 DiabetesPatienten [41] mit diastolischen Blutdruckzielwerten $<80 \mathrm{~mm} \mathrm{Hg}$ (erreichter Zielwert betrug $81 \mathrm{~mm} \mathrm{Hg}$ ) eine $51 \%$ Senkung der CV-Ereignisse im Vergleich zur Kontrollgruppe (< $85 \mathrm{~mm} \mathrm{Hg}$ or $<90 \mathrm{~mm} \mathrm{Hg})$. In der UKPDS-Studie [42] führte die Senkung des diastolischen Blutdruckes von 87 auf $82 \mathrm{~mm} \mathrm{Hg} \mathrm{zu}$ einer signifikanten Abnahme von Myokardinfarkt (-21\%) und Schlaganfall (-44\%). In der SAVOR-Studie [43] fand sich die niedrigste CV-Ereignisrate bei diastolischen Blutdruckwerten zwischen 80 und $90 \mathrm{~mm} \mathrm{Hg}$ und systolischen Blutdruckwerten bei $130-140 \mathrm{~mm}$ $\mathrm{Hg}$. Diastolische Blutdruckwerte $<60 \mathrm{~mm} \mathrm{Hg}$ waren mit einem erhöhten Myokard-Infarkt-Risiko assoziiert (HR 2,30 [95\% CI 1,50-3,53]) im Vergleich zu Patienten mit höheren diastolischen Blutdruckwerten (80-90 mm Hg). Die rezenten ESH/ESC Guidelines [32] empfehlen diastolische Blutdruckwerte von $<80-70$.

\section{Therapie mit Antihypertensiva}

Bei bestätigter Hypertonie (Blutdruck $\geq 140 / 90 \mathrm{~mm}$ $\mathrm{Hg}$ bei mehreren Messungen an verschiedenen Tagen) sollte eine medikamentöse Blutdrucktherapie begonnen und zeitnahe angepasst werden. Liegt der Blutdruck $\geq 160 / 100 \mathrm{~mm} \mathrm{Hg}$, so sollte bereits initial eine Kombination von zwei antihypertensiven Wirkstoffen gegeben werden [44]. Es sollten Antihypertensiva verwendet werden, für die bei Diabetes eine Reduktion von kardiovaskulären Ereignissen gezeigt wurde: ACE-Hemmer, Angiotensin-2-Rezeptorblocker, Diuretika vom Thiazid-Typ und Calciumantagonisten vom Dihydropyridin-Typ. Während von verschiedenen Wissenschaftsgesellschaften unterschiedliche Blutdruckzielwerte für Patienten mit Diabetes mellitus empfohlen werden, besteht bezüglich der Bevorzugung von ACE-Inhibitoren und Angiotensin-2Rezeptorblockern Übereinstimmung (Tab. 1).

Bei Patienten mit erhöhter Albuminausscheidung (Albumin/Kreatinin-Ratio $\geq 30 \mathrm{mg} / \mathrm{g}$ ) sollte ein ACEHemmer oder ein Angiotensin-Rezeptorblocker(ARBs) in der höchsten für die Hypertoniebehandlung zugelassenen und verträglichen Dosierung gegeben werden. Dies gilt nicht nur für hypertensive Patienten: Unabhängig vom Blutdruck sollten ARBs oder ACEHemmer bei Patienten verwendet werden, die eine erhöhte Albuminausscheidung aufweisen. Die Blutdruckzielwerte müssten allerdings entsprechend dem Alter, dem Pulsdruck, einer vorbestehenden koronaren Herzerkrankung, einer PAVK, Risiko der Progression der Nierenerkrankung sowie Abwesenheit oder Vorliegen einer diabetischen Retinopathie individualisiert werden.

Unter Behandlung mit einem ACE-Hemmer, einem Angiotensin-2-Rezeptorblocker oder einem Diuretikum sollte die Nierenfunktion zumindest einmal im Jahr kontrolliert werden.

Bei Diabetes-Patienten mit Nephropathie ist es besonders schwierig die Blutdruckzielwerte $\mathrm{zu}$ erreichen, sodass bei der Mehrzahl aller Diabetes-Patienten eine antihypertensive Kombinationstherapie erforderlich ist [44].

Die Kombination von ACE-Hemmern und Angiotensin-Rezeptorblockern ist zur Blutdrucksenkung nicht sinnvoll, eine Kombination dieser beiden Medikamentenklassen mit direkten Renin-Inhibitoren ist kontraindiziert. Es wurde in der Vergangenheit versucht durch eine Doppelblockade des Renin-Angiotensin-Aldosteron-Systems die Progression der Albuminurie besser zu beeinflussen als mit einer Monotherapie mittels ACE-Hemmer, ARB oder ReninInhibitoren. Bei diesen Studien (ONTARGET, ALTITUDE) zeigte sich zwar eine stärkere Reduktion der Albuminurie durch die duale Blockade, kardiovaskuläre und renale Ereignisse sowie schwere Nebenwirkungen (unter anderem Hypotonie und Hyperkaliämie) wurden allerdings unter dieser Kombinationstherapie signifikant häufiger beobachtet [45-47]. Aufgrund die- 
ser Daten empfehlen die 2012 publizierten KDIGOGuidelines, eine duale Blockade mittels ACE-Inhibitoren und ARB oder ARB plus Renin-Inhibitoren bei Diabetes-Patienten mit chronischer Nierenerkrankung bzw. Albuminurie nicht mehr einzusetzen [48]. Auch bei diesen Studien waren wahrscheinlich RRWerte deutlich unter $130 \mathrm{~mm} \mathrm{Hg}$ systolisch für das Auftreten von schwerwiegenden Ereignissen, sowie dem schlechteren Outcome bei Patienten mit zusätzlicher KHK verantwortlich.

Lange Zeit wurden Kombinationstherapien von ACE-Inhibitoren oder ARB mit niedrigdosierten Diuretika favorisiert. In der ACCOMPLISH-Studie [49] wurde allerdings bei 6000 Patienten mit Typ 2 Diabetes und Hypertonie nachgewiesen, dass bei gleichen Blutdruckzielwerten (135/80 mm Hg) die Kombination von ACE-Inhibitoren mit Kalziumantagonisten der Kombination von ACE-Inhibitoren und Diuretika deutlich überlegen war. Die Überlegenheit könnte aber auch darauf zurückzuführen sein, dass die Halbwertszeit von Amlodipin wesentlich länger ist als jene von Hydrochlorothiazid. Da Diabetes-Patienten häufig - insbesondere, wenn sie mit Insulin behandelt sind - eine vermehrte Wasserretention aufweisen, ist eine zusätzliche Therapie mit niedrigdosierten Diuretika im Sinne einer Dreifachkombination häufig sinnvoll.

In kontrollierten Interventionsstudien gelang es trotz Einsatz von 3 bis 4 Antihypertensiva in den meisten Fällen allerdings nicht, die Blutdruckzielwerte bei Patienten mit Diabetes und Hypertonie $\mathrm{zu}$ erreichen [44]. Noch ungünstiger war es, wenn Patienten mit diabetischer Nephropathie in Studien inkludiert wurden [44].

In einer Blutdruckzielwert-Studie für die Sekundärprävention an 29.000 Patienten mit Typ 2 Diabetes in Deutschland und Österreich [50] erreichten $67 \%$ der Patienten nach Herzinfarkt den Blutdruckzielwert 130/80 mm Hg und $90 \%$ der Patienten nach Schlaganfall den Zielwertbereich 120/70-140/90. In einer rezenten kanadischen Studie erreichten nur $36 \%$ der von praktischen Ärzten betreuten Diabetes-Patienten den Zielwert von $<130 / 80 \mathrm{~mm} \mathrm{Hg}$ [51].

Mancia und Grassi [4] berichteten, dass in prospektiven randomisierten Studien bei hypertensiven Diabetes-Patienten nur in 5 von 13 Studien systolische Blutdruckzielwerte $\leq 140$ und nur in 6 von 13 Studien diastolische Blutdruckzielwerte $\leq 80 \mathrm{~mm} \mathrm{Hg}$ erreicht wurden.

Bei Patienten mit inadäquat kontrollierten Blutdruckwerten trotz einer Dreifachkombination antihypertensiver Wirkstoffe, die auch ein Diuretikum einschließt, kann die zusätzliche Gabe eines Mineralokortikoidantagonisten zur Blutdruckkontrolle erwogen werden. Unter dieser Kombinationstherapie sind allerdings regelmäßige Kontrollen der Nierenfunktion und des Kaliums unabdingbar. Gegenwertig wird die blutdrucksenkende Wirkung der Mineralkortikoidantagonisten in mehreren Studien auf eine Beeinflus-
sung/Verbesserung des kardiovaskulären und/oder renalen Event-freien Überleben bei Patienten mit Diabetes/diabetischer Nephropathie geprüft.

\section{Blutdrucksenkung durch Antidiabetika}

Bei Verwendung von mehreren Antidiabetika-Klassen kommt es zu einer signifikanten Blutdrucksenkung, wodurch die Blutdruckeinstellung der Patienten verbessert wird. Während unter Metformin, Insulin, Sulfonylharnstoffen und DPP-4-Inhibitoren keine Veränderung der Blutdruckwerte beobachtet wurde, senken SGLT-2-Inhibitoren, GLP-1 Rezeptoragonisten und Pioglitazon den Blutdruck signifikant unabhängig davon, ob eine Hypertension oder eine Normotension vorliegt.

In einer Metaanalyse von Qayyum [52] an 13 Studien senkten die Glitazone im Vergleich zu Placebo den systolischen/diastolischen Blutdruck signifikant um 4,9/1,8 mm Hg.

In einer Metaanalyse von Wang et al. [53] wurde der blutdrucksenkende Effekt von GLP-1 Rezeptoragonisten in 13 randomized controlled trials (RCTs) analysiert. Exenatide senkte im Vergleich zu Placebo den systolischen/diastolischen Blutdruck um 5,2 und 5,4 mm Hg und Liraglutid senkte den systolischen Blutdruck um 5,6 mm Hg.

In einer Metaanalyse von Baker et al. [54] an 6 Studien, in denen unter einer Therapie mit SGLT2-Inhibitoren 24-Stunden Blutdruckmessungen durchgeführt wurden, fanden sich signifikante Blutdrucksenkungen systolisch um 3,76 mm Hg und diastolisch um 1,83 mm Hg. Die Blutdrucksenkung während des Tages betrug 4,3 mm Hg und während der Nacht 2,6 mm Hg. Die Blutdrucksenkungen waren unter den 4 getesteten SGLT-2-Inhibitoren (Canagliflozin, Dapagliflozin, Empagliflozin und Ertogliflozin) nicht signifikant unterschiedlich und korrelierten auch nicht mit dem Ausgangsblutdruck oder der Gewichtsabnahme [54]. Der blutdrucksenkende Effekt der SGLT-2-Inhibitoren ist bezüglich der Nephroprotektion und Kardioprotektion prinzipiell als günstig zu beurteilen.

In der EMPA REG Outcome Studie [55] und in der CANVAS-Studie [56] waren die Ausgangsblutdrucke bei 135/77 mm Hg und 136/78 mm Hg, da ca. $80-90 \%$ der Patienten mit einem oder mehreren Antihypertensiva behandelt wurden. Erwartungsgemäß sank der Blutdruck in beiden Studien unter den SGLT-2Inhibitoren signifikant weiter ab. In der EMPA-REG Outcome Studie kam es unter Empagliflozin zu einer hochsignifikanten Senkung von kardiovaskulärem Tod, Gesamtmortalität, und Progression der Nierenerkrankung, der Blutdruckabfall von BP 135,3/76,6 mm $\mathrm{Hg}$ auf 131,3/75,1 mm $\mathrm{Hg}$ hat keinerlei negativen Effekt. Diese Daten weisen darauf hin, dass Blutdruckwerte um $130 \mathrm{~mm} \mathrm{Hg}$ auch bei Hochrisikopatienten mit Typ 2 Diabetes nicht gefährlich sein dürften. Bei älteren Patienten sollte bei einer Kombinationstherapie von Antihypertensiva und SGLT-2-Inhibitoren 


\begin{tabular}{|c|c|c|}
\hline Empfehlungen & Klasse $^{\mathrm{a}}$ & Evidenz $^{\mathrm{b}}$ \\
\hline $\begin{array}{l}\text { Bei Patienten mit diabetischer oder nicht-diabetischer chronischer } \\
\text { Nierenerkrankung wird empfohlen, einen Blutdruck von } \geq 140 / 90 \mathrm{~mm} \\
\mathrm{Hg} \text { durch Lebensstilmodifikation und Blutdruck-senkende } \\
\text { Medikamente zu behandeln. }\end{array}$ & $\mathbf{I}$ & A \\
\hline \multirow{2}{*}{$\begin{array}{l}\text { Bei Patienten mit diabetischer oder nicht-diabetischer chronischer } \\
\text { Nierenerkrankung: } \\
\text { - Wird empfohlen, den systolischen Blutdruck auf einen Zielbereich } \\
\text { zwischen } 130-139 \mathrm{~mm} \mathrm{Hg} \mathrm{zu} \mathrm{senken.} \\
\text { - Eine individualisierte Behandlung sollte je nach Verträglichkeit und } \\
\text { Wirkung auf Nierenfunktion und Elektrolyte erwogen werden. }\end{array}$} & $\mathbf{I}$ & A \\
\hline & Ila & C \\
\hline $\begin{array}{l}\text { RAS-Blocker sind effektiver zur Senkung von Albuminurie als andere } \\
\text { Antihypertensiva, und werden als Teil der Behandlungsstrategie bei } \\
\text { hypertensiven Patienten mit Mikroalbuminurie oder Proteinurie } \\
\text { empfohlen. }\end{array}$ & $\mathbf{I}$ & A \\
\hline $\begin{array}{l}\text { Die Kombination von RAS-Blockern mit Kalziumkanalblockern oder } \\
\text { einem Diuretikumc wird als initiale Therapie empfohlen. }\end{array}$ & $\mathbf{I}$ & A \\
\hline Die Kombination zweier RAS-Blocker wird nicht empfohlen. & III & A \\
\hline
\end{tabular}

Abb. 1 TherapeutischeStrategien zur Behandlung von Bluthochdruck bei Vorliegen einer chronischen Nierenerkrankung. RAS Renin-Angiotensin System; 'aEmpfehlungsklasse; bEvidenzstufe; "bei geschätzter glomerulärer Filtrationsrate $<30 \mathrm{ml} / \mathrm{min} / 1.73 \mathrm{~m}^{2}$ kein Thiazid /Thiazid-ähnliches Diuretikum verwenden, wenn nötig Schleifendiuretikum erwägen. (Aus Williams et al. [33]. Reproduced and translated by permission of Oxford University Press on behalf of the Euro-

eine häufigere Blutdruckmessung erfolgen, um im Bedarfsfall die Zahl oder Dosis der Antihypertensiva $\mathrm{zu}$ reduzieren.

\section{Blutdruckziele bei Diabetes mellitus je nach Ko- morbiditäten}

\section{Blutdruckzielwerte und antihypertensive Therapie bei alten Patienten mit Diabetes}

Es existieren nur wenige Studien, die spezielle ältere Patienten mit Diabetes mellitus eingeschlossen haben, obwohl 30-60\% aller Diabetes-Patienten in der Welt älter als 65 Jahre sind [57]. Ältere Patienten stellen eine sehr heterogene Gruppe dar, viele Patienten zwischen 65-75 Jahren sind nicht selten frei von Komplikationen, generell ist aber die Komorbidität bei diesen Patienten deutlich erhöht (vor allem Herzinsuffizienz und Nephropathie). Bei komplexen und gebrechlichen Patienten sollten etwas höhere Zielwerte $(<150 / 90 \mathrm{~mm} \mathrm{Hg})$ angestrebt werden, bei Patienten ohne Komplikationen empfehlen die Guidelines der ADA und IDF Zielwerte von 140/85, jene von Kanada allerdings $<130 / 80 \mathrm{~mm} \mathrm{Hg}$ [57]. Die rezenten ESH/ESC Guidelines [32] empfehlen hingegen systolische Blutdruckwerte $<140-120$ für Patienten mit einem Alter von 65-79 Jahren und idente Werte auch für Patienten $>80$ Jahre. Aufgrund der zunehmenden Inzidenz pean Society of Cardiology. All rights reserved. (C) European Society of Cardiology. For permissions, please email journals.permissions@oup.com. Please visit: www.escardio.org. OUP and ESC are not responsible or in any way liable for the accuracy of the translation. Guntram Schernthaner is solely responsible for the translation in this publication. This figure is not included under the Creative Commons CC BY license of this publication)

von Herzinsuffizienz sollten Diuretika in der Kombinationstherapie bei diesen Patienten nicht fehlen.

\section{Nephropathie}

Eine internationale Expertengruppe hat für KDIGO (Kidney Disease Improving Global Outcomes) evidenzbasierte Richtlinien für die antihypertensive Behandlung von Diabetes-Patienten mit chronischer Nierenerkrankung oder Makroalbuminurie festgelegt, die 2012 publiziert wurden [48]. Für Patienten mit chronischer Nierenerkrankung und Diabetes mellitus ohne erhöhte Albuminausscheidung wurden Blutdruck-Zielwerte < 140/90 mm Hg empfohlen. Bei Patienten, die zusätzlich eine erhöhte Albuminausscheidung aufweisen, wurden Zielwerte von $<130 / 80 \mathrm{~mm}$ Hg empfohlen [48].

Verschiedene Studien haben gezeigt, dass differente Blutdruckzielwerte unterschiedliche Effekte auf Endorganschäden ausüben [10, 17]. Während möglichst niedrige Blutdruckwerte für die Schlaganfallprävention [17] und zur Verhinderung der Progression der diabetischen Nephropathie [10] sehr günstig sein dürften, werden kardiovaskuläre Ereignisse insgesamt oder auch die Sterblichkeit dadurch nicht gesenkt. Unter besonders niedrigen Blutdruckwerten (sowohl systolisch als auch diastolisch) wurden sogar signifikant vermehrt kardiovaskuläre Ereignisse beobachtet, sodass von niedrigeren Zielwerten als $130 / 80 \mathrm{~mm}$ 


\begin{tabular}{|l|c|c|}
\hline Empfehlungen & Klasse $^{\mathbf{a}}$ & Evidenz $^{\mathbf{b}}$ \\
\hline $\begin{array}{l}\text { Eine medikamentöse antihypertensive Therapie wird empfohlen bei } \\
\text { Patienten mit Diabetes und einem Blutdruck } \geq 140 / 90 \mathrm{mmHg} .\end{array}$ & I & A \\
\hline $\begin{array}{l}\text { Bei Patienten mit Diabetes, die Blutdruck-senkende Medikamente } \\
\text { erhalten wird empfohlen: }\end{array}$ & I & A \\
$\begin{array}{l}\text { Zielwert systolischer Blutdruck: } 130 \mathrm{mmHg} \text { und }<130 \mathrm{mmHg} \\
\text { sofern toleriert, jedoch nicht }<120 \mathrm{mmHg} . \\
\text { Bei älteren Patienten ( } \geq 65 \text { Jahre), Zielbereich für den systolischen } \\
\text { Blutdruck } 130-139 \mathrm{mmHg} .\end{array}$ & I & A \\
\cline { 2 - 3 } $\begin{array}{l}\text { Zielbereich diastolischer Blutdruck: }<80 \mathrm{mmHg} \text {, jedoch nicht }<70 \\
\text { mmHg. }\end{array}$ & I & C \\
\hline $\begin{array}{l}\text { Es wird empfohlen, die Behandlung mit einer Kombination von RAS- } \\
\text { Blockern mit Kalziumkanalblockern bzw. Thiazid / Thiazid-ähnlichem } \\
\text { Diuretikumc zu beginnen. }\end{array}$ & I & A \\
\hline $\begin{array}{l}\text { Die gleichzeitige Gabe zweier RAS-Blocker, z.B. ein ACE-Hemmer und } \\
\text { ARB, ist nicht indiziert. }\end{array}$ & III & A \\
\hline
\end{tabular}

Abb. 2 Behandlungsstrategien des Hochdrucks bei $\mathrm{Pa}-$ tienten mit Diabetes. RAS Renin-Angiotensin System, ACE Angiotensin-Converting-Enzyme, ARB Angiotensin-Rezeptorblocker; ${ }^{a}$ Empfehlungsklasse; bevidenzstufe; ${ }^{\mathrm{c}}$ bei geschätzter glomerulärer Filtrationsrate $<30 \mathrm{ml} / \mathrm{min} / 1.73 \mathrm{~m}^{2}$ kein Thiazid/Thiazid-ähnliches Diuretikum verwenden, wenn nötig Schleifendiuretikum erwägen. (Aus Williams et al. [33]. Reproduced and translated by permission of Oxford University

Hg im Allgemeinen abzuraten ist. In der ROADMAPStudie [58] wurde etwa mittels einer aggressiven Blutdrucksenkung (Zielblutdruck $<130 / 80 \mathrm{~mm} \mathrm{Hg}$ ) das Auftreten einer Mikroalbuminurie zwar signifikant verzögert, gleichzeitig stiegen aber die fatalen kardiovaskulären Ereignisse signifikant an, wobei dies primär auf vermehrte kardiovaskuläre Todesfälle bei Patienten mit präexistenter KHK zurückzuführen war.

In einer rezenten Metanalyse von 24 RCTs an nahezu 70.000 Patienten (14 Studien mit Diabetes und 10 Studien ohne Diabetes) wurde unter einer Blutdrucksenkung von $10 / 5 \mathrm{~mm} \mathrm{Hg}$ eine hochsignifikante Reduktion der terminalen Niereninsuffizienz (ESRD) um $21 \%$ nachgewiesen mit einer Number-Neededto-Treat (NNT) von nur 8 Patienten bei einer Therapiedauer von 5 Jahren [59]. Bemerkenswert ist allerdings der hochsignifikante Unterschied $(p<0,0001)$ zwischen Patienten mit Diabetes (HR 0,79 (0,66-0,95)) und ohne Diabetes (HR 1,01 $(0,81-1,26)$. Diese Metaanalyse würde also nahelegen, dass eine Blutdrucksenkung bei Nichtdiabetikern keine Senkung des Risikos einer terminalen Niereninsuffizienz bewirkt, während bei Patienten mit Diabetes mellitus eine nur relativ kleine Blutdrucksenkung von 10/5 mm Hg eine dramatische Verbesserung bewirken könnte.

Allerdings waren sowohl in ACCORD-BP als auch in SPRINT-Studie [39] Patienten im intensivierten Arm einem höheren Risiko für das Entstehen einer CNI $\left(\right.$ eGFR $>30 \%$ auf $<60 \mathrm{~mL} / \mathrm{min}$ per $1,73 \mathrm{~m}^{2}$ ) ausgesetzt, als im Standard-Arm. Eine Überwachung der Nierenfunktion unter intensivierter Blutdruckkontrolle erscheint daher sehr wichtig. Die therapeutischen
Press on behalf of the European Society of Cardiology. All rights reserved. (C) European Society of Cardiology. For permissions, please email journals.permissions@oup.com. Please visit: www.escardio.org. OUP and ESC are not responsible or in any way liable for the accuracy of the translation. Guntram Schernthaner is solely responsible for the translation in this publication. This figure is not included under the Creative Commons CC BY license of this publication)

Strategien zur Behandlung von Bluthochdruck bei Vorliegen einer chronischen Nierenerkrankung, wie sie vor kurzem in den neuen ESC/ESH Guidelines empfohlen wurden [33], sind in der Abb. 1 zusammengefasst.

\section{Schlaganfall}

Xie at el [60]. analysierten den Effekt einer Blutdrucksenkung auf das Schlaganfall-Risiko in einer Network Analyse aus 21 RCTs, die insgesamt 65.000 Patienten eingeschlossen hatten. Untersucht wurde die Risikosenkung einer Blutdrucksenkung von 10/5 mm Hg bei systolischen Ausgangsblutdrucken >140, <140, >130 und $<130 \mathrm{~mm}$ Hg. Für Patienten mit den niedrigsten Ausgangsblutdrucken $(<130 \mathrm{~mm} \mathrm{Hg})$ fand sich noch immer eine signifikante Schlaganfall-Reduktion bei Diabetes-Patienten (HR 0,61 [95\%CI: 0,47-0,79]).

In der ACCORD-BP Studie [17] wurde unter der intensivierten Blutdrucksenkung mit einem Zielwert von $119,5 \mathrm{~mm} \mathrm{Hg}$ im Vergleich zum Kontrollarm $(133,5 \mathrm{~mm} \mathrm{Hg})$ ebenfalls eine hochsignifikante Risikoreduktion des Schlaganfalls (HR 0,59 [95\% CI: 0,39-0,89]) beobachtet.

\section{PAVK}

Diabetes-Patienten mit PAVK haben eine weitaus schlechtere Prognose als Diabetes-Patienten nach Herzinfarkt oder Schlaganfall, wie in der PROactive-Studie nachgewiesen werden konnte [61]. Früher wurde für PAVK-Patienten generell Blutdruckzielwer- 
Tab. 2 Individuelle Blutdruckzielwerte für Patienten mit Diabetes mellitus in Abhängigkeit von der Komorbidität und Alter

\begin{tabular}{|c|c|c|}
\hline Patientenspezifika & Blutdruckzielwert & Kommentar \\
\hline Keine Komorbidität & $<140 / 85 \mathrm{~mm} \mathrm{Hg}$ & Benefit wurde bis $130 / 80$ beobachtet, darunter Risikozunahme möglich \\
\hline Junge Patienten & $130 / 80 \mathrm{~mm} \mathrm{Hg}$ & Benefit vor kurzem gezeigt \\
\hline Alte Patienten & $<150 / 85 \mathrm{~mm} \mathrm{Hg}$ & Zielwert hängt von den Komorbiditäten und Ihrer Komplexität ab \\
\hline Nephropathie & \multirow[t]{2}{*}{$<140 / 90 \mathrm{~mm} \mathrm{Hg}$} & \multirow[t]{2}{*}{-} \\
\hline Ohne Albuminurie & & \\
\hline Nephropathie & \multirow[t]{2}{*}{$<130 / 80 \mathrm{~mm} \mathrm{Hg}$} & \multirow[t]{2}{*}{ Benefit am größten bei $120 / 80 \mathrm{~mm} \mathrm{Hg}$} \\
\hline Mit Albuminurie & & \\
\hline Schlaganfall & $120 / 80 \mathrm{~mm} \mathrm{Hg}$ & Patienten mit gleichzeitiger KHK $130 / 80 \mathrm{~mm} \mathrm{Hg}$ \\
\hline PAVK & $<145 / 90 \mathrm{~mm} \mathrm{Hg}$ & $\begin{array}{l}\text { Bei kritischer Extremitätenischämie können passager höhere Blutdruckwerte } \\
\text { notwendig sein (bis zur Revaskularisierung) }\end{array}$ \\
\hline KHK & $130 / 80 \mathrm{~mm} \mathrm{Hg}$ & $\begin{array}{l}\text { Zu niedrige diastolische Blutdruckwerte }(<70 \mathrm{~mm} \mathrm{Hg}) \text { sind mit einer verkürz- } \\
\text { ten Lebenserwartung assoziiert }\end{array}$ \\
\hline
\end{tabular}

te $<130 / 80$ mm Hg gefordert, obwohl dafür keine Evidenz aus Studien vorlag. In der posthoc Analyse der INVEST-Studie [62] wurde das besonders hohe Risiko der PAVK-Patienten (Diabetesanteil 41\%) bestätigt. Bei insgesamt 2699 Patienten mit PAVK (1106 Patienten mit Typ 2 Diabetes) zeigte sich nach einer Studiendauer von 2,6 Jahren ein enger Zusammenhang zwischen den Blutdruckwerten und dem primären Endpunkt (Gesamtmortaliät, nichtfataler Herzinfarkt und nichtfataler Schlaganfall). Der beste Outcome (niedrigste HR für den primären Outcome) fand sich bei systolischen Blutdruckwerten zwischen 135 bis $145 \mathrm{~mm} \mathrm{Hg}$ und diastolischen Werten zwischen 60-90 mm Hg. Bei niedrigeren systolischen Blutdruckwerten stieg die HR bis auf 1,7 (systolischer Blutdruck um $110 \mathrm{~mm} \mathrm{Hg)} \mathrm{an,} \mathrm{bei} \mathrm{Blutdruckwerten}$ um $180 \mathrm{~mm} \mathrm{Hg}$ lag die HR sogar bei 2,5 [62]. Insgesamt ist die Datenlage bei PAVK und Diabetes mellitus besonders schwierig, da es wenig Primärstudien gibt.

\section{KHK}

Verschiedene Studien zeigen, dass insbesondere bei Diabetes-Patienten mit gleichzeitig bestehender KHK das Blutdruckziel nicht $<130 \mathrm{~mm} \mathrm{Hg}$ systolisch liegen sollte. So zeigt etwa eine rezente Auswertung von Daten der SAVOR TIMI 53 Studie [43] das niedrigste kardiovaskuläre Risiko bei Blutdruckwerten von $130-140 \mathrm{~mm} \mathrm{Hg}$ systolisch und von $80-90 \mathrm{~mm} \mathrm{Hg}$ diastolisch. Da allerdings sehr viele Diabetes-Patienten diese Blutdruckzielwerte trotz Einsatz mehrerer Antihypertensiva bei weitem nicht erreichen, ist im klinischen Alltag die Angst vor zu niedrigen Blutdruckwerten wahrscheinlich wesentlich unbedeutender als das Bemühen systolische Blutdruckwerte unter $140 \mathrm{~mm} \mathrm{Hg}$ zu erreichen.

\section{Rezente Metaanalysen und Expertenmeinungen}

In den letzten beiden Jahren sind mehrere Metaanalysen von weltführenden Experten auf dem Gebiet der Hypertensiologie publiziert wurden. Die zum Teil widersprüchlichen Ergebnisse machen es verständ- lich, warum verschiedene Wissenschaftsgesellschaften unterschiedliche Blutdruckzielwerte empfehlen. Aggarwal et al. [63] analysierten den Effekt von Blutdruckzielwerten $<120 / 80$ mm Hg versus $<140 / \mathrm{mm} \mathrm{Hg}$ an 14.000 Patienten mit und ohne Diabetes aus den kontrollierten Studien von ACCORD-PB und SPRINT. Patienten mit den niedrigen Blutdruckzielwerten hatten signifikant weniger CV-Ereignisse (HR 0,83; $95 \%$ CI, 0,74-0,92; $P<0,001$, eine Interaktion mit Diabetes war nicht nachweisbar, wenngleich die positiven Effekte einer intensiven Blutdrucksenkung bei den $\mathrm{Pa}-$ tienten ohne Diabetes viel stärker ausgeprägt waren. Thomopolous et al. [59] analysierten das Ausmaß einer Blutdrucksenkung von 10/5 mm Hg in 27 RCTs bei Patienten mit Diabetes und in 28 RCTS bei Patienten ohne Diabetes in Abhängigkeit vom Ausgangsblutdruck $>140,130-140$ und <130 systolisch. Während die Blutdrucksenkung auch bei niedrigen Ausgangsblutdrucken bei Nichtdiabetikern für alle Endpunkte positive Effekt zeigte, fand sich dies bei DiabetesPatienten nur für den Endpunkt Schlaganfall, ein signifikanter Anstieg von Gesamtmortalität und kardiovaskulärem Tod wurde allerdings nicht beobachtet. Aus einer weiteren Metaanalyse hat dieselbe Arbeitsgruppe [64] den Schluss gezogen, dass Blutdruckwerte um $130 \mathrm{~mm} \mathrm{Hg}$ mit dem geringsten vaskulären Risiko assoziiert sein dürften, eine ganz ähnliche Beurteilung kommt von der Arbeitsgruppe von Sarafidis [65]. Die rezente umfangreiche Analyse von Mancia und Grassi [4] schließt mit der Feststellung, dass die Blutdruckzielwerte bei Diabetes-Patienten wahrscheinlich niedriger als $<140 / 90$ sein sollten und wahrscheinlich 130/80 mm Hg betragen sollten. Die Evidenz für Blutdruckzielwerte $<130 / 80 \mathrm{~mm} \mathrm{Hg}$ ist nach Meinung dieser Experten nicht ausreichend, Blutdruckzielwerte $<120 / 80 \mathrm{~mm} \mathrm{Hg}$ sollten aber unbedingt vermieden werden.

Die Empfehlungen der rezent publizierten ESC/ESH Guidelines [33] für die Therapie der Hypertonie bei Patienten mit Diabetes mellitus sind in der Abb. 2 zusammengefasst. 


\section{Individualisierung der Blutdruckzielwerte bei Dia- betes-Patienten in Abhängigkeit von der vorlie- genden Komorbidität bzw. Alter des Patienten}

Ähnlich wie bei der Blutzuckereinstellung erscheint es aufgrund der publizierten kontrollierten Studien sinnvoll, eine Individualisierung der Blutdruckzielwerte in Abhängigkeit vom Alter und der Komorbidität (Schlaganfall, Herzinfarkt, Herzinsuffizienz, PAVK, Nephropathie) anzustreben, wie in der Tab. 2 dargestellt.

\section{Zusammenfassung}

Internationale Wissenschaftsgesellschaften empfehlen für Diabetes-Patienten unterschiedliche Blutdruckzielwerte $<140 / 90 \mathrm{~mm} \quad \mathrm{Hg}$ (ADA) oder $<130 / 80 \mathrm{~mm} \mathrm{Hg}$ (AHA, ESH/ESC). Wichtig erscheint es, dass vor allem die konservativen Zielwerte $<140 / 90 \mathrm{~mm} \mathrm{Hg}$ von allen Patienten erreicht werden. Patienten mit bestimmter Komorbidität - nach Schlaganfall oder bei Vorliegen einer Nephropathie profitieren allerdings eindeutig von niedrigeren Zielwerte, sodass es aufgrund der gegenwärtigen Evidenz am sinnvollsten erscheint, eine Individualisierung der Blutdruckeinstellung bei Patienten mit Diabetes mellitus anzustreben. Ein Zielblutdruck um 130/80 mm Hg dürfte nach gegenwärtiger Evidenz jener sein, der mit der relativ besten Prävention von vaskulären Komplikationen und mit der größten Sicherheit einhergeht. Niedrigere Zielwerte $(120 / 80 \mathrm{~mm} \mathrm{Hg})$ sind für die Primär-und Sekundärprävention von Schlaganfall und Nephropathie prinzipiell günstig, wegen des erhöhten Auftretens von schwerwiegenden Nebenwirkungen ist allerdings Vorsicht angezeigt. Blutdruckzielwerte unter $120 \mathrm{~mm} \mathrm{Hg}$ systolisch und unter 70 diastolisch können aufgrund der potentiellen Steigerung der Mortalität keineswegs empfohlen werden.

Funding Open access funding provided by Medical University of Vienna.

Interessenkonflikt C.H. Saely hat in Bezug auf diesen Artikel von folgenden Unternehmen, die teils auch fördernde Mitglieder der ÖDG sind, Forschungsunterstützungen und/ oder Honorare erhalten: MSD, Merck, AstraZeneca, Novartis, Pfizer, Genericon, Takeda, Bayer, Roche. H. Drexel: hat in Bezug auf diesen Artikel von folgenden Unternehmen, die teils auch fördernde Mitglieder der ÖDG sind, Forschungsunterstützungen und/oder Honorare erhalten: Amgen, AstraZeneca, Bayer Austria, Boehringer-Ingelheim, Meda Pharma, Merck Sharp \& Dohme, Novo Nordisk, sanofi-aventis, Servier. G. Schernthaner, G.-H. Schernthaner und B. Watschinger geben an, dass kein Interessenkonflikt besteht.

Open Access Dieser Artikel wird unter der Creative Commons Namensnennung 4.0 International Lizenz (http:// creativecommons.org/licenses/by/4.0/deed.de) veröffentlicht, welche die Nutzung, Vervielfältigung, Bearbeitung, Verbreitung und Wiedergabe in jeglichem Medium und Format erlaubt, sofern Sie den/die ursprünglichen Autor(en) und die Quelle ordnungsgemäß nennen, einen Link zur Creative
Commons Lizenz beifügen und angeben, ob Änderungen vorgenommen wurden.

Hinweis des Verlags Der Verlag bleibt in Hinblick auf geografische Zuordnungen und Gebietsbezeichnungen in veröffentlichten Karten und Institutsadressen neutral.

\section{Literatur}

1. Muntner P, Carey RM, Gidding S, Jones DW, Taler SJ, Wright JT Jr, Whelton PK. Potential U.S. Population impact of the 2017 ACC/AHA high blood pressure guideline. JAm Coll Cardiol. 2018;71(2):109-18.

2. Bakris G, Sorrentino M. Redefining hypertension-assessing the new blood-pressure guidelines. N Engl J Med. 2018;378(6):497-9.

3. Messerli FH, Bangalore S. Lowering the thresholds of diseases: is anyone still healthy? J Am Coll Cardiol. 2018;71(2):119-21.

4. ManciaG, GrassiG. Blood pressuretargetsintype2 diabetes. Evidence against or in favour of an aggressive approach. Diabetologia. 2018;61(3):517-25.

5. Schernthaner G, Drexel H, Rosenkranz A, Schernthaner $\mathrm{GH}$, Watschinger B. Antihypertensive therapy in diabetes mellitus: Guidelines of the Austrian Diabetes Association 2016. Wien Klin Wochenschr. 2016;128(Suppl2):S62-S7.

6. Schernthaner G, Drexel H, Rosenkranz AR, Schernthaner GH, Watschinger B, Austrian Diabetes Association. Antihypertensive therapyin diabetes mellitus-2012 guidelines of the Austrian Diabetes Association. Wien Klin Wochenschr. 2012;124(Suppl2):23-7.

7. Ferrannini E, Cushman WC. Diabetes and hypertension: the bad companions. Lancet. 2012;380(9841):601-10.

8. Wing RR, Lang W, Wadden TA, Safford M, Knowler WC, Bertoni AG, Hill JO, Brancati FL, Peters A, Wagenknecht L, Look AHEAD Rsearch Group. Benefits of modest weight loss in improving cardiovascular risk factors in overweight and obese individuals with type 2 diabetes. Diabetes Care. 2011:34:1481-6.

9. Stamler J, Vaccaro O, Neaton JD, Wentworth D. Diabetes, other risk factors, and 12-yr cardiovascular mortality for men screned in the Multiple Risk Factor Intervention Trial. Diabetes Care. 1993;16(2):434-44.

10. Adler AI, Stratton IM, Neil HA, Yudkin JS, Matthews DR, Cull CA, Wright AD, Turner RC, Holman RR. Association of systolic blood pressure with macrovascular and microvascular complications of type 2 diabetes (UKPDS 36): prospective observational study. BMJ.2000;321(7258):412-9.

11. Pohl MA, Blumenthal S, Cordonnier DJ, De Alvaro F, Deferrari G, Eisner G, Esmatjes E, Gilbert RE, Hunsicker LG, de Faria JB, Mangili R, Moore J Jr, Reisin E, Ritz E, Schernthaner G, Spitalewitz S, Tindall H, Rodby RA, Lewis EJ. Independent and additive impact of blood pressure control and angiotensin II receptor blockade on renal outcomes in the irbesartan diabetic nephropathy trial: clinical implications and limitations. JAm Soc Nephrol. 2005;16(10):3027-37.

12. Mancia G, De Backer G, DominiczakA, Cifkova R, Fagard R, Germano G, Grassi G, Heagerty AM, Kjeldsen SE, Laurent S, Narkiewicz K, Ruilope L, Rynkiewicz A, Schmieder RE, Struijker Boudier HA, Zanchetti A, Vahanian A, Camm J, De Caterina R, Dean V, Dickstein K, Filippatos G, FunckBrentano C, Hellemans I, Kristensen SD, McGregor K, Sechtem U, Silber S, Tendera M, Widimsky P, Zamorano JL, Kjeldsen SE, Erdine S, Narkiewicz K, Kiowski W, AgabitiRosei E, Ambrosioni E, Cifkova R, Dominiczak A, Fagard R, Heagerty AM, Laurent S, Lindholm LH, Mancia G, Manolis 
A, Nilsson PM, Redon J, Schmieder RE, Struijker-Boudier HA, Viigimaa M, Filippatos G, Adamopoulos S, AgabitiRosei E, Ambrosioni E, Bertomeu V, Clement D, Erdine S, Farsang C, Gaita D, Kiowski W, Lip G, Mallion JM, Manolis AJ, Nilsson PM, O'Brien E, Ponikowski P, Redon J, Ruschitzka F, Tamargo J, van Zwieten P, Viigimaa M, Waeber B, Williams B, Zamorano JL, The task force for the management of arterial hypertension of the European Society of Hypertension, The task force for the management of arterial hypertension of the European Society of Cardiology. 2007 Guidelines for the management of arterial hypertension: The Task Force for the Management of Arterial Hypertension of the European Society of Hypertension (ESH) and of the European Society of Cardiology (ESC). Eur Heart J. 2007;28(12):1462-536.

13. Wang J, Geiss LS, Cheng YJ, Imperatore G, Saydah SH, James C, Gregg EW. Long-term and recent progress in blood pressure levels among U.S. adults with diagnosed diabetes, 1988-2008. Diabetes Care. 2011;34(7):1579-81. https:// doi. org/10.2337/dc11-0178.

14. Mancia G,LaurentS,Agabiti-RoseiE,AmbrosioniE, Burnier M, Caulfield MJ, Cifkova R, Clément D, Coca A, Dominiczak A, Erdine S, Fagard R, Farsang C, Grassi G, Haller H, Heagerty A, Kjeldsen SE, Kiowski W, Mallion JM, Manolis A, Narkiewicz K, Nilsson P, Olsen MH, Rahn KH, Redon J, Rodicio J, Ruilope L, Schmieder RE, Struijker-Boudier HA, van Zwieten PA, Viigimaa M,ZanchettiA, European Society ofHypertension. Reappraisal of European guidelines on hypertension management: a European Society of Hypertension Task Force document. J Hypertens. 2009;27(11):2121-58.

15. Deedwania PC. Blood pressure control in diabetes mellitus: is lower always better, and how low should itgo? Circulation. 2011;123(24):2776-8.

16. Garcia-Touza M, Sowers JR. Evidence-based hypertension treatment in patients with diabetes. J Clin Hypertens. 2012;14(2):97-102.

17. Kalaitzidis RG, Bakris GL. Pros and cons of aggressive blood pressure lowering in patients with type 2 diabetes. Curr Vasc Pharmacol.2012;10(2):156-61.

18. ACCORD Study Group, Cushman WC, Evans GW, Byington RP, Goff DC Jr, Grimm RH Jr, Cutler JA, Simons-Morton DG, Basile JN, Corson MA, Probstfield JL, Katz L, Peterson KA, Friedewald WT, Buse JB, Bigger JT, Gerstein HC, IsmailBeigi F. Effects of intensive blood-pressure control in type 2 diabetes mellitus. NEngl J Med. 2010;362(17):1575-85.

19. Buckley LF, Dixon DL, Wohlford GF 4th, Wijesinghe DS, Baker WL, Van Tassell BW. Intensive Versus Standard Blood Pressure Cntrol in SPRINT-Eligible Participants of ACCORD-BP. Diabetes Care. 2017;40(12):1733-8.

20. Brouwer TF, Vehmeijer JT, Kalkman DN, Berger WR, van den Born BH, Peters RJ, Knops RE. Intensive blood pressure lowering in patients with and patients without type 2 diabetes: a pooled analysis from two randomized trials. Diabetes Care. 2018;41(6):1142-8.

21. Schernthaner G, Barnett AH, Betteridge DJ, Carmena R, Ceriello A, Charbonnel B, Hanefeld M, Lehmann R, Malecki MT, Nesto R, Pirags V, Scheen A, Seufert J, Sjohölm A, Tsatsoulis A, DeFronzo R. Is the ADA/EASD algorithm for the management of type 2 diabetes (January 2009) based on evidence or opinion? A critical analysis. Diabetologia. 2010;53(7):1258-69.

22. de Boer IH, Bakris G, Cannon CP. Individualizing blood pressure targets for people with diabetes and hypertension: comparing the ADA and the ACC/AHA recommendations. JAMA. 2018;319(13):1319-20.

23. Cooper-DeHoff RM, Gong Y, Handberg EM, Bavry AA, Denardo SJ, Bakris GL, Pepine CJ. Tight blood pressure control and cardiovascular outcomes among hypertensive patients with diabetes and coronary artery disease. JAMA. 2010;304(1):61-8.

24. Anderson RJ, Bahn GD, Moritz TE, Kaufman D, Abraira C, Duckworth W, VADT Study Group. Blood pressure and cardiovascular disease risk in the Veterans Affairs Diabetes Trial. Diabetes Care. 2011;34(1):34-8.

25. American Diabetes Association. 9. Cardiovascular disease and risk management: standards of medical care in diabetes-2018. Diabetes Care. 2018;41(suppl 1):S86-S104.

26. Whelton PK, Carey RM, Aronow WS, Casey DE Jr, Collins KJ, Dennison Himmelfarb C, de Palma SM, Gidding S, Jamerson KA, Jones DW, MacLaughlin EJ, Muntner P, Ovbiagele B, Smith SC Jr, Spencer CC, Stafford RS, Taler SJ, Thomas RJ, Williams KA Sr, Williamson JD, Wright JT Jr.. 2017 ACC/AHA/AAPA/ABC/ACPM/AGS/APhA/ASH/ ASPC/NMA/PCNA Guideline for the Prevention, Detection, Evaluation, and Management of High Blood Pressure in Adults: A Report of the American College of Cardiology/ American Heart Association Task Force on Clinical Practice Guidelines. J Am Coll Cardiol. 2017;71(19):e127-e248.

27. Daskalopoulou SS, Rabi D, Zamke KB, CHEP for the Canadian Hypertension Education Program. The 2015 Canadian Hypertension Education Program recommendations for blood pressure measurement, diagnosis, assessment of risk, prevention, and treatment of hypertension. Can J Cardiol. 2015;31(5):549-69.

28. International Diabetes Federation Clinical Guidelines Task Force.. Global guideline for type 2 diabetes. Brussels: IDF; 2012.

29. World Health Organization. Aglobal brief on hypertension. Geneva:WHO;2013.

30. Krause T, Lovibond K, Caulfield M, McCormack T, Williams B. Management of hypertension: summary of NICE guidance. BMJ.2011;343:d4891.

31. Shimamoto K, Ando K, Fujita T, et al. The Japanese Society of Hypertension guidelines for the management of hypertension (JSH 2014). Hypertens Res. 2014;37(4):253-390.

32. Association of Physicians of India. Indian guidelines on hypertension (IGH) - III. 2013. J Assoc Physicians India. 2013;61(2 Suppl):6-36.

33. Williams B, Mancia G, SpieringW, Agabiti Rosei E, Azizi M, BurnierM, Clement DL, Coca A, de Simone G, Dominiczak A, Kahan T, Mahfoud F, Redon J, Ruilope L, Zanchetti A, Kerins M, Kjeldsen SE, Kreutz R, Laurent S, Lip GYH, McManus R, Narkiewicz K, Ruschitzka F, SchmiederRE, Shlyakhto E, Tsioufis C, Aboyans V, Desormais I, ESC/ESH Guidelines for the management of arterial hypertension. Eur Heart J. 2018;39(33):3021-3104. https://doi.org/10.1093/ eurheartj/ehy339

34. James PA, Oparil S, Carter BL, Cushman WC, DennisonHimmelfarb C, Handler J, Lackland DT, LeFevre ML, MacKenzie TD, Ogedegbe O, Smith SC Jr, Svetkey LP, Taler SJ, Townsend RR, WrightJTJr, Narva AS, OrtizE. 2014 evidencebased guideline for the management of high blood pressure in adults: report from the panel members appointed to the Eighth Joint National Committee (JNC 8). JAMA. 2014;311(5):507-20.

35. Action to Control Cardiovascular Risk in Diabetes Study Group, Gerstein HC, Miller ME, Byington RP, Goff DC Jr, Bigger JT, Buse JB, Cushman WC, Genuth S, IsmailBeigi F, Grimm RH Jr, Probstfield JL, Simons-Morton DG, FriedewaldWT. Effects of intensive glucoselowering in type 2 diabetes. NEngl J Med. 2008;358(24):2545-59.

36. SPRINT Research Group, Wright JT Jr, Williamson JD, Whelton PK, Snyder JK, Sink KM, Rocco MV, Reboussin DM, Rahman M, OparilS, Lewis CE, Kimmel PL, Johnson KC, GoffDC Jr, Fine LJ, Cutler JA, Cushman WC, Cheung AK, Ambrosius 
WT.ARandomized Trial of Intensiveversus Standard BloodPressure Control. NEnglJ Med. 2015;373(22):2103-16.

37. Lamprea-Montealegre JA, de Boer IH. Reevaluating the evidence for blood pressure targets in type 2 diabetes. Diabetes Care. 2018;41(6):1132-3.

38. Margolis KL, O'Connor PJ, Morgan TM, Buse JB, Cohen RM, Cushman WC, Cutler JA, Evans GW, Gerstein HC, Grimm RH Jr, Lipkin EW, Narayan KM, Riddle MC Jr, Sood A, Goff DC Jr.. Outcomes of combined cardiovascular risk factor management strategies in type 2 diabetes: the ACCORD randomized trial. Diabetes Care. 2014;37(6):1721-8.

39. Beddhu S, Greene T, Boucher R, Cushman WC, Wei G, Stoddard G, IxJH, Chonchol M, Kramer H, CheungAK, Kimmel PL, Whelton PK, Chertow GM. Intensive systolic blood pressure control and incident chronic kidney disease in people with and without diabetes mellitus: secondary analyses of two randomised controlled trials. Lancet Diabetes Endocrinol. 2018;6(7):555-63.

40. Wan EYF, Yu EYT, Chin WY, Fung CSC, Fong DYT, Choi EPH, Chan AKC, Lam CLK. Effect of achieved systolic blood pressure on cardiovascular outcomes in patients with type 2 diabetes: a population-based retrospective cohort study. Diabetes Care. 2018;41(6):1134-41.

41. Hansson L, Zanchetti A, Carruthers SG, Dahlöf B, Elmfeldt D, Julius S, Ménard J, Rahn KH, Wedel H, Westerling S. Effects of intensive blood-pressure lowering and low-dose aspirin in patients with hypertension: principal results of the Hypertension Optimal Treatment (HOT) randomised trial.HOT Study Group. Lancet. 1998;351(9118):1755-62.

42. UK Prospective Diabetes Study Group. Tight blood pressure control and risk of macrovascular and microvascular complications in type 2 diabetes: UKPDS 38. BMJ. 1998;317(7160):703-13.

43. Bergmark BA, Scirica BM, Steg PG, Fanola CL, Gurmu Y, Mosenzon O, Cahn A, Raz I, Bhatt DL, SAVOR-TIMI 53 Investigators. Blood pressure and cardiovascular outcomes in patients with diabetes and high cardiovascular risk. Eur Heart J.2018;39(24):2255-62.

44. Schernthaner G, Schernthaner GH. Hypertension and diabetes: need for combination therapy. In: Goldstein BJ, Müller-Wieland D, Hrsg. Type 2 diabetes. Principle and practice. 2. Aufl. New York: Informa Healthcare; 2007.

45. Mann JF, Schmieder RE, McQueen M, Dyal L, Schumacher H, Pogue J, Wang X, Maggioni A, Budaj A, Chaithiraphan S, Dickstein K, Keltai M, Metsärinne K, Oto A, Parkhomenko A, Piegas LS, Svendsen TL, Teo KK, Yusuf S, ONTARGET investigators. Renal outcomes with telmisartan, ramipril, or both, in people at high vascular risk (the ONTARGET study): a multicentre, randomised, double-blind, controlled trial. Lancet. 2008;372(9638):547-53.

46. Redon J, Mancia G, Sleight P, Schumacher H, Gao P, Pogue J, Fagard R, Verdecchia P, Weber M, Böhm M, Williams B, Yusoff K, Teo K, Yusuf S, ONTARGET Investigators. Safety and efficacy of low blood pressures among patients with diabetes: subgroup analyses from the ONTARGET (ONgoing Telmisartan Alone and in combination with Ramipril Global Endpoint Trial). J Am Coll Cardiol. 2012;59(1):7483.

47. Angeli F, Reboldi G, Mazzotta G, Poltronieri C, Garofoli M, Ramundo E, Biadetti A, Verdecchia P. Safety and efficacy of aliskiren in the treatment of hypertension and associated clinical conditions. Curr Drug Saf. 2012;7(1):76-85.

48. KDIGOBloodPressureWorkGroup. KDIGOclinicalpractice guideline for the management of blood pressure in chronic kidney disease. Kidney Int. 2012;2(Suppl):337-414.

49. Weber MA, Bakris GL, Jamerson K, Weir M, Kjeldsen SE, Devereux RB, Velazquez EJ, Dahlöf B, Kelly RY, Hua TA, He- ster A, Pitt B, ACCOMPLISH Investigators. Cardiovascular events during differing hypertension therapies in patients with diabetes. JAm Coll Cardiol. 2010;56(1):77-85.

50. Bohn B, Schöfl C, Zimmer V, Hummel M, Heise N, Siegel E, Karges W, Riedl M, Holl RW, DPV-initiative. Achievement of treatmnt goals for secondary prevention of myocardial infarction or stroke in 29,325 patients with type 2 diabetes: a German/Austrian DPV-multicenter analysis. Cardiovasc Diabetol.2016;15:72.

51. Grenier J, Goodman SG, Leiter LA, Langer A, Teoh H, Bhatt DL, Cheng AYY, Tan MK, Ransom T, Connelly KA, Yan AT. Blood pressure management in adults with type 2 diabetes: insights from the diabetes mellitus status in Canada (DMSCAN) survey. Can J Diabetes. 2018;42:130-7.

52. Adumaityte QRJ. A meta-analysis of the effect of thiazolidinediones on blood pressure. JClin Hypertens. 2006;8:1928.

53. WangB,ZhongJ, LinH, ZhaoZ, YanZ, HeH, NiY, LiuD, ZhuZ. Blood pressure-lowering effects of GLP-1 receptor agonists exenatide and liraglutide: a meta-analysis of clinical trials. Diabetes Obes Metab. 2013;15(8):737-49.

54. BakerWL, Buckley LF, KellyMS, BucheitJD, ParodED, Brown $\mathrm{R}$, Carbone S, Abbate Dixon DL. Effects of sodium-glucose cotransporter 2 inhibitors on 24-hour ambulatory blood pressure: a systematic review and meta-analysis. JAm Heart Assoc. 2017;6:e5686.

55. Zinman B, Wanner C, LachinJM, FitchettD, BluhmkiE, Hantel S, Mattheus M, Devins T, Johansen OE, Woerle HJ, Broedl UC, Inzucchi SE, EMPA-REG OUTCOME Investigators. Empagliflozin, cardiovascular outcomes, and mortality in type 2. Diabetes. NEnglJ Med. 2015;373:2117-28.

56. Neal B, Perkovic V, Mahaffey KW, de Zeeuw D, Fulcher G, Erondu N, Shaw W, Law G, Desai M, Matthews DR, CANVAS Program Collaborative Group. Canagliflozin and cardiovascular and renal events in type 2 diabetes. $\mathrm{N}$ Engl J Med.2017;377:644-57.

57. Schernthaner G, Schernthaner-Reiter MH. Diabetes in the older patient: heterogeneity requires individualization of therapeutic strategies. Diabetologia. 2018;61:1503-16.

58. Haller H, Ito S, Izzo JL Jr, Januszewicz A, Katayama S, Menne J, Mimran A, Rabelink TJ, Ritz E, Ruilope LM, Rump LC, Viberti G, ROADMAP Trial Investigators. Olmesartan for the delay or prevention of microalbuminuria in type 2 diabetes. NEngl J Med.2011;364(10):907-17.

59. Thomopoulos C, Parati G, Zanchetti A. Effects of bloodpressure-lowering treatment on outcome incidence in hypertension: 10 - Should blood pressure management differ in hypertensive patients with and without diabetes mellitus? Overview and meta-analyses of randomized trials. JHypertens. 2017;35(5):922-44.

60. Xie XX, Liu P, Wan FY, Lin SG, Zhong WL, Yuan ZK, Zou JJ, Liu LB. Blood pressure lowering and stroke events in type 2 diabetes: a network meta-analysis of randomized controlled trials. Int JCardiol. 2016;208:141-6.

61. Dormandy JA, Betteridge DJ, Schernthaner G, Pirags V, Norgren L, PROactive investigators. Impact of peripheral arterial disease in patients with diabetes - results fromPROactive (PROactive 11). Atherosclerosis. 2009;202(1):272-81.

62. BavryAA, Anderson RD, GongY, DenardoSJ, Cooper-Dehoff RM, Handberg EM, Pepine CJ. Outcomes Among hypertensive patients with concomitant peripheral and coronary artery disease: findings from the International VErapamilSR/Trandolapril STudy. Hypertension. 2010;55(1):48-53.

63. Aggarwal R, Steinkamp J, Chiu N, Petrie B, Mirzan H. Intensive blood pressure targets for diabetic and other highrisk populations: a pooled individual patient data analysis. Hypertension. 2018;71(5):833-9. 
64. Thomopoulous C, Parati G, Zanchetti A. Effects of bloodpressure-lowering treatment on outcome incidence in hypertension. 11. Effects of total cardiovascular risk and achieved blood pressure: overview and meta-analyses of randomized trials. J Hypertens. 2017;35:2138-49.
65. Papadopoulou E, Angeloudi E, Karras S, Sarafidis P. The optimal blood pressure target in diabetes mellitus: a quest coming to an end? J Hum Hypertens. 2018; https:// doi.org/ 10.1038/s41371-018-0079-5. 